\title{
Quasi-analytic solution of the magnetotelluric vertical layered anisotropic model
}

\author{
Bing Xue, Ling Huang, Guangyou Fang and Yicai Ji \\ Key Laboratory of Electromagnetic Radiation and Sensing Technology \\ Chinese Academy of Sciences 100190 \\ Beijing, China \\ xuebing14@mails.ucas.ac.cn
}

\begin{abstract}
In order to explain the surveyed data more accurately and have a better understanding of the distribution of geoelectricity, two-dimensional partial differential equations of Magnetotelluric in anisotropic media are employed to establish vertical layered medium axially anisotropic model by Fourier Transform method. The quasi-analytic solutions of the electromagnetic field distribution of the TM mode are derived from the media of three vertical layers. The MT responses of isotropic media degenerated from anisotropic media are analyzed and compared with the results computed by finite element method. Similarly, the MT responses of anisotropic media are calculated to analyze the characters of response functions. This model has a further guiding significance on cognizing the magnetotelluric response characteristics of the complicated vertical layered medium model and explaining the detected data.
\end{abstract}

Keywords-magnetotelluric method; anisotropic model; quasianalytic solution; finite element method; response

\section{INTRODUCTION}

For the isotropic media model of stratum has become unable to explain the explored data accurately and the anisotropic medium model can reflect the geoelectric structure in a better way, it is necessary to introduce the aniotropic medium model ${ }^{[1]}$. At present, there are a lot of researches of the MT in the numerical simulation and the inversion method of the horizontal layered medium models, which mainly concentrate on the isotropic medium. For the limits of the ability of the field observation, the computing resource and the development of the inversion method, the study of the threedimensional model is still relatively little and the practical applications are limited ${ }^{[2]}$. Hence, the one-dimensional along with the two-dimensional model is still the main model for the study of the stratigraphic texture ${ }^{[3]}$.

When the deepness of the layer near the ground level reaches two or three times of surface effect depth of the electromagnetic wave, the electromagnetic wave in the layer far from the ground level can be ignored ${ }^{[4]}$. Hence, the research on the vertical layered geoelectric model meets the actual situation. At present, there are only a few articles about the analytic-solutions of the MT response of anisotropic media $^{[4]}$, so in order to explain the detected data and the geoelectric structure more accurately, we present a mathematical model for the vertical layered anisotropic media. In this paper, firstly the quasi-analytic solutions of vertical three-layered anisotropic media model are deduced. Then the accuracy of the isotropic model degenerated from the anisotropic model is verified using finite element method. Finally, the MT responses of isotropic and anisotropic media are obtained and the response functions are discussed.

\section{THEORETICAL MODEL}

\section{A. Theoretical Model}

Suppose the zero point is at the ground plane, then the $\mathrm{x}$ axis and the $y$ axis are derived form the ground plane, and the $\mathrm{z}$ axis is perpendicular to the ground. If the coupling effect between the electric field and the magnetic field caused by the anisotropy is ignored, the anisotropic conductivity tensor (angular anisotropic model) can be written as:

$$
\bar{\sigma}=\left[\begin{array}{ccc}
\sigma_{x} & 0 & 0 \\
0 & \sigma_{y} & 0 \\
0 & 0 & \sigma_{z}
\end{array}\right]
$$

The TE and the TM mode are expressed as partial differential equations of second order, which are the diffusion field propagation equations of no electromagnetic mutual coupling effects.

$$
\begin{aligned}
& \frac{1}{\sigma_{z}} \frac{\partial^{2} H_{x}}{\partial y^{2}}+\frac{1}{\sigma_{y}} \frac{\partial^{2} H_{x}}{\partial z^{2}}=-j \omega \mu H_{x} \\
& -\frac{1}{j \omega \mu} \frac{\partial^{2} E_{x}}{\partial y^{2}}-\frac{1}{j \omega \mu} \frac{\partial^{2} E_{x}}{\partial z^{2}}=\sigma_{x} E_{x}
\end{aligned}
$$




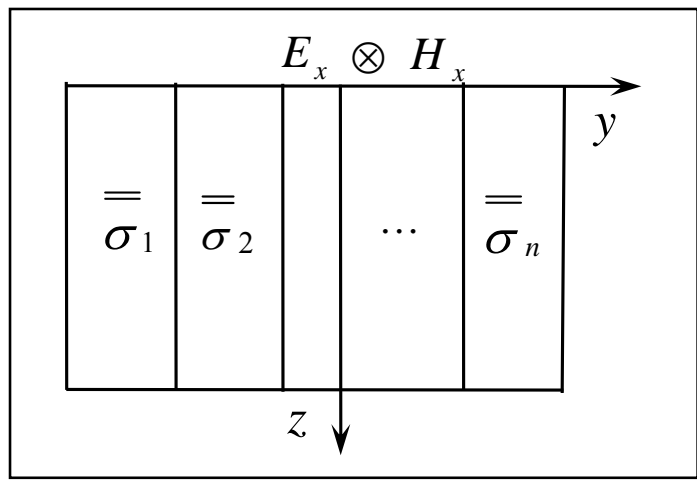

Fig. 1. The half space vertical layered anisotropic media model

\section{B. TM Mode}

The equation of magnetic field $H_{x}$ can be obtained by (2) and the boundary conditions of calculating regional. Permeability can be expressed as $\mu=\mu_{0}=4 \pi \times 10^{7} \mathrm{H} \mathrm{m}^{-1}$. The incident wave is the approximated plane wave. When $z=0, H_{x}^{i}=G$. The $G$ is the constant. The $i$ expresses the media of the $i$ layers. The electromagnetic wave can be attenuated by the stratum. When $z \rightarrow \infty, H_{x}^{i}, E_{y}^{i}$ and $E_{z}^{i} \rightarrow 0$. Fourier sine transform acts on the (2) in the $z$ axis. The spectral domain equation can be expressed as:

$$
\frac{1}{\sigma_{z}} \frac{d^{2} \widetilde{H}_{x}^{i}}{d y^{2}}+\frac{1}{\sigma_{y}} \widetilde{\varphi}^{i}=-j \omega \mu \widetilde{H}_{x}^{i}
$$

Where, $\widetilde{H}_{x}^{i}$ and $\widetilde{\varphi}^{i}$ are the Fourier sine transformation equations of $H_{x}^{i}$ and $\frac{d^{2} H_{x}^{i}}{d z^{2}}$, respectively. Using The integration by parts method and boundary conditions, $\tilde{\varphi}^{i}$ can be written as:

$$
\begin{aligned}
& \widetilde{\varphi}^{i}=\int_{0}^{\infty} \frac{d^{2} H_{x}^{i}}{d z^{2}} \sin \xi z d z=\left.\sin \xi z \frac{d H_{x}^{i}}{d z}\right|_{0} ^{\infty} \\
& -\left.\xi \cos \xi z \cdot H_{x}^{i}\right|_{0} ^{\infty}-\xi^{2} \widetilde{H}_{x}^{i}=\xi G-\xi^{2} \widetilde{H}_{x}^{i}
\end{aligned}
$$

Then, define $P_{i}^{2}=\frac{\sigma_{x}^{i}}{\sigma_{y}^{i}}\left(\xi^{2}-j \omega \mu \sigma_{y}^{i}\right)$. For the $N$ layers model, $H_{x}^{i}$ can be written as:

$$
\left\{\begin{aligned}
\tilde{H}_{x}^{1} & =C_{1} e^{P_{1} y}+U_{1} \\
\widetilde{H}_{x}^{2} & =C_{2} e^{P_{2} y}+D_{2} e^{-P_{2} y}+U_{2} \\
& \vdots \\
\widetilde{H}_{X}^{N-1} & =C_{N-1} e^{P_{N-1} y}+D_{N-1} e^{-P_{N-1} y}+U_{N-1} \\
\widetilde{H}_{x}^{N} & =D_{N} e^{-P_{N} y}+U_{N}
\end{aligned}\right.
$$

where, $U_{i}=\frac{\sigma_{z}^{i} \xi G}{\sigma_{y}^{i} P_{i}^{2}}$. The magnetic field on the boundary of each layer can be expressed as $H_{x}^{i}\left(y_{i}, z\right)=H_{x}^{i+1}\left(y_{i}, z\right)=f_{i}(z) \cdot f_{i}(z)$ is the magnetic field function of the fracture surface of $i \cdot y_{i}$ is the $\mathrm{y}$ axis coordinate value.

$$
\begin{gathered}
\tilde{H}_{x}^{1}=\left(\tilde{f}_{1}-U_{1}\right) e^{P_{1}\left(y-y_{i}\right)}+U_{1} \\
\tilde{H}_{x}^{i}=C_{i}\left(\tilde{f}_{1}, \tilde{f}_{2}, \ldots \tilde{f}_{N}\right) e^{P_{i} y}+D_{i}\left(\tilde{f}_{1}, \tilde{f}_{2}, \ldots \tilde{f}_{N}\right) e^{-P_{i} y}+U_{i}
\end{gathered}
$$

$$
(i=2,3, \cdots, N-1)
$$

$$
\widetilde{H}_{x}^{N}=\left(\tilde{f}_{N-1}-U_{N}\right) e^{-P_{3}\left(y-y_{N-1}\right)}+U_{N}
$$

$\tilde{f}_{i}$ is Fourier sine transformation expression of $f_{i}(z)$ in the $Z$ axis.

$$
E_{x}^{i}\left(y_{i}, z\right)=E_{x}^{i+1}\left(y_{i}, z\right)
$$

$N-1$ expressions about $\widetilde{f}_{i}$ can be obtained. Then, we can get $\tilde{f}_{i}$. Fourier sine inverse transformation acts on (7-9):

$$
H_{x}^{1}=G e^{j \sqrt{j \omega \mu \sigma_{y}^{1}} z}+\frac{2}{\pi} \int_{0}^{\infty}\left(\tilde{f}_{1}(\xi)-U_{1}\right) e^{P_{1}(y+d)} \sin \xi z d \xi
$$

$$
H_{x}^{i}=G e^{j \sqrt{j \omega \mu \sigma_{y}^{i} z}}+\frac{2}{\pi} \int_{0}^{\infty}\left(C_{i} e^{P_{i} y}+D_{i} e^{-P_{i} y}\right) \sin \xi z d \xi
$$

$$
(i=2,3, \cdots, N-1)
$$




$$
\begin{aligned}
H_{x}^{N} & =G e^{j \sqrt{j \omega \mu \sigma_{y}^{i} z}} \\
& +\frac{2}{\pi} \int_{0}^{\infty}\left(\widetilde{f}_{N-1}(\xi)-U_{N}\right) e^{-P_{N}\left(y-y_{i}\right)} \sin \xi z d \xi
\end{aligned}
$$

According to the Residue theorem: $F^{-1}\left\{U_{i}\right\}=e^{j \sqrt{j \omega \mu \sigma_{y}^{i}} z}$. Here we consider a simple model of multilayer media. Let $N=3, y_{1}=-d, y_{2}=d$.

$$
\begin{gathered}
H_{x}^{1}=G e^{j \sqrt{j \omega \mu \sigma_{y}^{1}} z}+\frac{2}{\pi} \int_{0}^{\infty}\left(\tilde{f}_{1}(\xi)-U_{1}\right) e^{P_{1}(y+d)} \sin \xi z d \xi \\
H_{x}^{2}=G e^{j \sqrt{j \omega \mu \sigma_{y}^{2}} z}+\frac{2}{\pi} \int_{0}^{\infty}\left(C_{2} e^{P_{2} y}+D_{2} e^{-P_{2} y}\right) \sin \xi z d \xi \\
C_{2}=\frac{\left(\widetilde{f}_{2}-U_{2}\right) e^{2 P_{2} d}-\left(\tilde{f}_{1}-U_{2}\right)}{e^{3 P_{2} d}-e^{-P_{2} d}} \\
D_{2}=\frac{\left(\tilde{f}_{1}-U_{2}\right) e^{2 P_{2} d}-\left(\tilde{f}_{2}-U_{2}\right)}{e^{3 P_{2} d}-e^{-P_{2} d}} \\
H_{x}^{3}=G e^{j \sqrt{j \omega \mu \sigma_{y}^{3} z}}+\frac{2}{\pi} \int_{0}^{\infty}\left(\tilde{f}_{2}(\xi)-U_{3}\right) e^{-P_{3}(y-d)} \sin \xi z d \xi(16) \\
\tilde{f}_{1}=\frac{\left(Q_{3}+T+S\right)\left(T U_{2}-S U_{2}\right)+S Q_{3} U_{3}+\left(Q_{3}+T\right) Q_{1} U_{1}}{\left(Q_{1}+T\right)\left(Q_{3}+T\right)-S^{2}}( \\
\tilde{f}_{2}=\frac{\left(Q_{1}+T+S\right)\left(T U_{2}-S U_{2}\right)+S Q_{1} U_{1}+\left(Q_{1}+T\right) Q_{3} U_{3}}{\left(Q_{1}+T\right)\left(Q_{3}+T\right)-S^{2}}(18)
\end{gathered}
$$

Here, $Q_{i}=\frac{P_{i}}{\sigma_{z}^{i}}, T=\frac{Q_{2}}{\tanh 2 P_{2} d}, S=\frac{Q_{2}}{\sinh 2 P_{2} d} \cdot \tanh (x)$ and $\sinh (x)$ are the hyperbolic tangent and hyperbolic sine function. $E_{y}^{i}$ is written as:

$$
\begin{aligned}
E_{y}^{1}= & G / \sigma_{y}^{1} j \sqrt{j \omega \mu \sigma_{y}^{1}} e^{j \sqrt{j \omega \mu \sigma_{y}^{1}} z} \\
& +\frac{2}{\pi \sigma_{y}^{1}} \int_{0}^{\infty}\left(\tilde{f}_{1}(\xi)-U_{1}\right) e^{P_{1}(y+d)} \xi \cos \xi z d \xi
\end{aligned}
$$

$$
\begin{aligned}
E_{y}^{2} & =G / \sigma_{y}^{2} j \sqrt{j \omega \mu \sigma_{y}^{2}} e^{j \sqrt{j \omega \mu \sigma_{y}^{2}} z} \\
& +\frac{2}{\pi \sigma_{y}^{2}} \int_{0}^{\infty}\left(C_{2} e^{P_{2} y}+D_{2} e^{-P_{2} y}\right) \xi \cos \xi z \xi
\end{aligned}
$$

$$
\begin{aligned}
E_{y}^{3}= & G / \sigma_{y}^{3} j \sqrt{j \omega \mu \sigma_{y}^{3}} e^{j \sqrt{j \omega \mu \sigma_{y}^{3}} z} \\
& +\frac{2}{\pi \sigma_{y}^{3}} \int_{0}^{\infty}\left(\tilde{f}_{2}(\xi)-U_{3}\right) e^{-P_{3}(y-d)} \xi \cos \xi z d \xi
\end{aligned}
$$

The conductivities of the media are isotropic and $\sigma^{i}=\sigma$, $H_{x}^{i}=G e^{j \sqrt{j \omega \mu \sigma} z}$. These results meet the diffusion effect solutions of the homogeneous half space. That is, the physical characteristics of the electromagnetic wave transmission are verified. And it also indicates that the electromagnetic wave tending to infinity of space displays the unified diffusion wave. The diffusion properties can also appear in the place far from the fracture surface, which is usually two or three times of electromagnetic wave surface effect depth ${ }^{[8]}$.

\section{TE Mode}

In this case, as it is only $\sigma_{x}$ in (3), this mode cannot reflect the characteristics of anisotropic media independently. However, we can still study the anisotropic media by the characteristics of the TE mode and the TM mode electromagnetic fields together. The derivations of the TE mode are similar to the TM's. The derivation process will not be repeated in this paper.

\section{MT Response Functions}

After the electromagnetic parameters of the TE mode and the TM mode are calculated, the characteristic impedance $Z_{T E}$ and $Z_{T M}$ of the TE mode and the TM mode are marked by calculating the ratio of the transverse electric fields and magnetic fields. Then, apparent resistivities and impedance phases can be obtained and the calculation formula can be expressed as:

$$
\rho_{T E, T M}=\left|Z_{T E, T M}\right|^{2} / \omega \mu
$$

$$
\phi_{T E, T M}=\tan ^{-1}\left(\operatorname{Im}\left[Z_{T E, T M}\right] / \operatorname{Re}\left[Z_{T E, T M}\right]\right)
$$

$\left|Z_{T E, T M}\right|$ is the module of the characteristic impedance. $\operatorname{Im}\left\{Z_{T E, T M}\right\}$ and $\operatorname{Re}\left\{Z_{T E, T M}\right\}$ are the imaginary and real parts of the characteristic impedance, respectively.

\section{MODEL ANALYSIS AND VERIFICATION}

Because of the continuous transverse currents in the interfaces, in the calculation of electromagnetic parameters, 
for TM model, $E_{y}^{i}$ in the interface of two-layered media are different. According to $\sigma_{y}^{i} E_{y}^{i}=\sigma_{y}^{i-1} E_{y}^{i-1}$, if the conductivities on both sides of fracture surface are different, $E_{y}^{i}$ are different, too. While, for the TE model, according to $\mu^{i} H_{y}^{i}=\mu^{i-1} H_{y}^{i-1}$, $H_{y}^{i}=H_{y}^{i-1}$ can be obtained. Therefore, the apparent resistivities of TM mode are intermittent, while the TE's are continuous $^{[8]}$.

We degenerate the anisotropic model to an isotropic one. Set $\sigma_{1}=0.1 \mathrm{~S} / \mathrm{m}, \sigma_{1} / \sigma_{2}=\sigma_{1} / \sigma_{3}=100, d=30 \mathrm{~km}$. The apparent resistivities and impedance phases results of the vertical layered model is shown in Fig.3. Near infinity, apparent resistivities of the TE mode and the TM mode tend to be consistent, which are expressed as the actual value of the resistivities of the uniform space. It shows that when there is no fracture surface, propagation properties of the TE and the $\mathrm{TM}$ are similar.

This paper also presents the calculated results by using finite element method. Comparing the apparent resistivities and impedance phases of these two methods, we can see that the solutions of the Fourier transform method are very consistent with those of the finite element method. This proves that the solutions are validated.

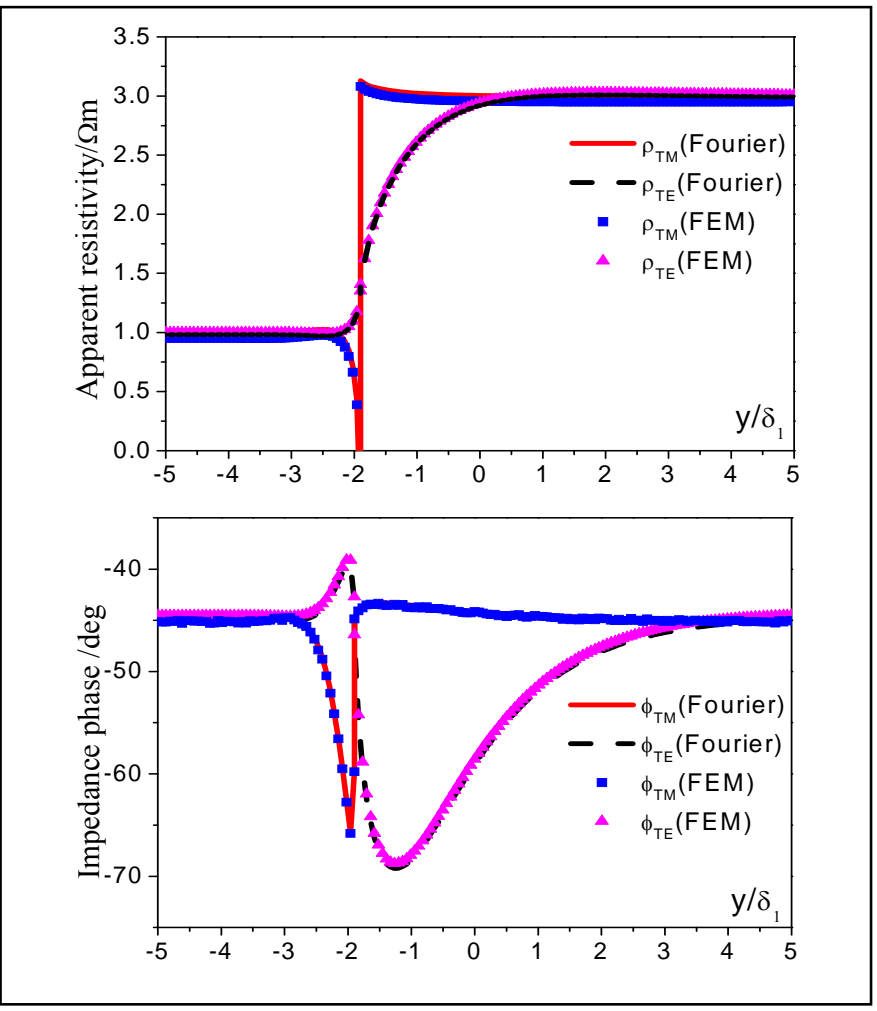

Fig. 2. Apparent resistivities and impedance phases of vertical layered anisotropic media

\section{Anisotropic Media Model}

Set $T=10 \mathrm{~s}, d=20 \mathrm{~km}$. Based on the above formulas, we can obtain the MT response functions of the anisotropic media. As shown in Fig.3, the TE mode can only reflect that the variation of the conductivities of the $\mathrm{x}$ axis has an effect on the apparent resistivities and the impedance phases of the media. When the ratio of the conductivities on both sides of the fracture surface is fixed in the TE mode, the smaller conductivities are, the greater the anomaly range of apparent resistivities and impedance phases are. Apparent resistivities of the TM mode are mainly represented as the conductivities in the $y$ axis direction, which is similar to isotropic media. Conductivities in the $\mathrm{z}$ axis mainly affect the distributions of the electromagnetic field on both sides of the fracture surface. The larger the value is, the greater the scope of the influence is. Close to infinity, the apparent resistivities of the TM mode tend to the conductivities in the y axis, while the TE's tend to the conductivities in the $\mathrm{x}$ axis, which reflects the distributions of anisotropic media on the horizontal direction.

\section{CONCLUSIONS}

The analysis of the results shows that the medium layer thickness has major influence on the MT responses of media. The MT responses of the TE mode are associated with the conductivities of the $\mathrm{x}$ axis, which cannot reflect the properties of the geoelectric anisotropic media. And the MT responses of the TM mode can reflect the profile of the anisotropic media properties. Therefore, in order to reflect the characteristics of geoelectric anisotropic media, the two mode (TE and TM) need to be considered together. In addition, in the TM mode, the conductivities of the $\mathrm{z}$ axis affect the MT responses at the location near the fracture surfaces. The actual observation data are not easy to characterize the conductivities of the $\mathrm{z}$ axis.

This MT two-dimensional vertical layered anisotropic media model built in this paper can reflect the geoelectric structure of the vertical layered media. And it is shown that the responses on the fracture surfaces are changed by the abnormal media, which also represents the anisotropic properties for the media. This model can explain the detected data well, which paves the way to recognize the MT response characteristics of the complicated vertical layered media model.

\section{REFERENCES}

[1] J.E. Mann, "The importance of anisotropic conductivity in magnetotelluric interpretation," J. Geophys. Res., 1965, vol. 70, pp. 2940-2942.

[2] M.A. Daniyan and W.J. Peeples, "Application of Rayleigh-FFT technique to three-dimensional Magnetotelluric interpretation," Annales Geophysicae, 1986, vol. 4B, pp. 441-456.

[3] J.T. Weaver, B.V. Lequang and G. Fischer, "A comparison of analytic and numerical results for a two-dimensional control model in electromagnetic induction. 1. B-polarization calculations," Geophys. J. Roy. Astron. Soc., 1985, Vol. 82, pp. 263-277.

[4] B.A. Hobbs, "Analytical solutions to global and local problems of electromagnetic induction in the Earth,” Phys. Earth Planet Inter., 1975, vol. 10 , pp. $250-261$. 


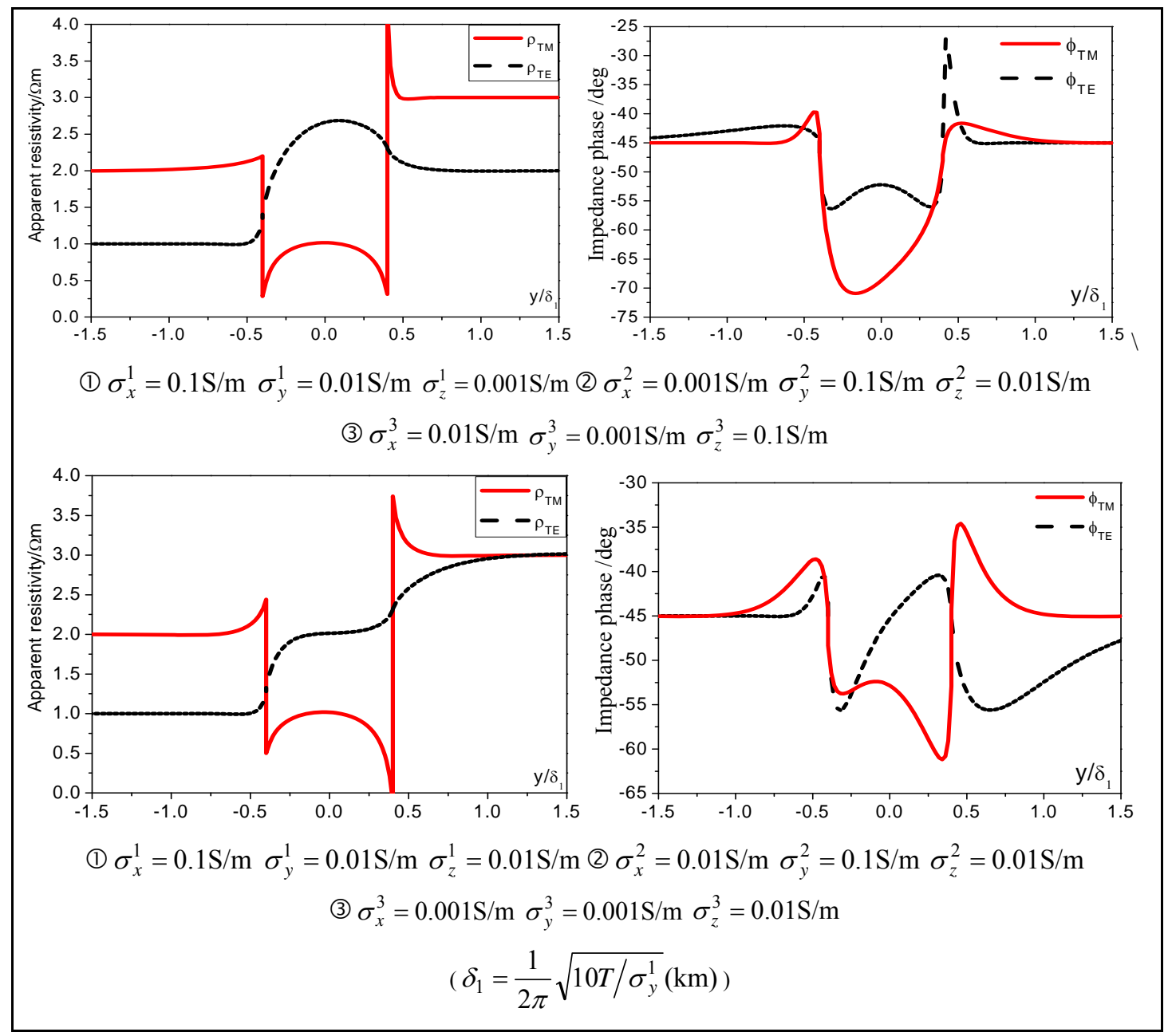

Fig. 3. Apparent resistivities and impedance phases of vertical layered anisotropic media 